\title{
A Multi-Unit Profit Competitive Mechanism for Cellular Traffic Offloading
}

\author{
Jun Wu, ${ }^{1}$ Yu Qiao, ${ }^{2}$ Lei Zhang, ${ }^{2}$ Chongjun Wang, ${ }^{2 *}$ Meilin Liu ${ }^{3}$ \\ ${ }^{1}$ Jiangsu Provincial Key Laboratory of E-Business, Nanjing University of Finance \& Economics, Nanjing 210003, China \\ ${ }^{2}$ State Key Laboratory for Novel Software Technology at Nanjing University, Nanjing 210023, China \\ ${ }^{3}$ Department of Computer Science and Engineering, Wright State University, Dayton, OH 45435, USA \\ jwucie@nufe.edu.cn, \{chjwang, lzhang\}@nju.edu.cn, meilin.liu@wright.edu
}

\begin{abstract}
Cellular traffic offloading is nowadays an important problem in mobile networking. We model it as a procurement problem where each agent sells multi-units of a homogeneous item with privately known capacity and unit cost, and the auctioneer's demand valuation function is symmetric submodular. Based on the framework of random sampling and profit extraction, we aim to design a prior-free mechanism which guarantees a profit competitive to the omniscient single-price auction. However, the symmetric submodular demand valuation function and 2-parameter setting present new challenges. By adopting the highest feasible clear price, we successfully design a truthful profit extractor, and then we propose a mechanism which is proved to be truthful, individually rational and constant-factor competitive in a fixed market.
\end{abstract}

\section{Introduction}

In recent years, global mobile data traffic has been explosively growing due to the proliferation and in-depth use of smart mobile devices and applications. According to a white paper newly updated by Cisco (Cisco 2019), global mobile data traffic reached 11.5 exabytes per month at the end of 2017, up from 6.7 exabytes per month at the end of 2016. Moreover, it had grown 17-fold from 2012 to 2017, grown $71 \%$ in 2017 , and would continue to increase another 7fold between 2017 and 2022. Such a surge of mobile traffic would certainly deteriorate existing cellular networks' service quality if it is not handled properly.

One solution is to offload the mobile data traffic onto fixed networks through Wi-Fi or femtocell. Compared with updating the cellular network's infrastructure or building more towers, cellular traffic offloading can ease the burden of cellular networks and enhance users' experience in a timely and economical manner (Balasubramanian, Mahajan, and Venkataramani 2010; Dong et al. 2014; Wang et al. 2015; Zhang et al. 2016), and it has already been widely applied in practice. According to Cisco (Cisco 2019), 54\% of total mobile data traffic was offloaded onto the fixed network through Wi-Fi or femtocell in 2017, thus mobile offload had

\footnotetext{
${ }^{*}$ Chongjun Wang is the corresponding author. Copyright (c) 2020, Association for the Advancement of Artificial Intelligence (www.aaai.org). All rights reserved.
}

exceeded cellular traffic by a significant margin; and nearly three-fifths of traffic $(59 \%)$ will be offloaded from cellular networks by 2022. So, it is of great application value to study how to optimally offload the cellular traffic.

In practice, such femtocell devices or WiFi hotspots are usually owned by third-party entities (agents) such as schools, restaurants, shopping malls, residences and so on. Shifting cellular traffic to these agents requires to consume their own resources (e.g., bandwidth, data quota, electricity, etc.), thus the agents need to be well motivated, and their costs, which are privately known by themselves, should be properly compensated (Zhang et al. 2016). Under such circumstances, finding a good offloading solution is no longer a conventional optimization problem. Instead, what we need is a well-designed mechanism, that generates an optimized procurement solution which approximates the optimal one in an ideal omniscient scenario. Essentially, this is an algorithmic mechanism design (Nisan and Ronen 2001) problem.

One common case is that the macrocell of a cellular service provider (CSP) is divided into several non-overlapping small regions. Each agent is located inside one region and can provide some units of offloading resources for this region each at a fixed unit cost, and each agent's capacity and unit cost is her private information. The agents bid their capacities and unit costs strategically to obtain utilities as high as possible. The CSP can purchase some units from the agents, and her valuation on the units is measured according to her improvement in service quality. The question then is how to decide the purchase quantity from each agent and the associated payment in order to maximize the CSP's profit. Basically, this is a novel prior-free optimal mechanism design problem characterized by the following features:

1) Multi-unit 2-parameter. Each agent sells multiple units of a homogeneous item with privately known capacity (inventory volume) and unit cost;

2) Local diminishing return (LDR) demand valuation. LDR is a superset of concave additivity (Chan and Chen 2014) and a subset of diminishing return (Chan and Chen 2014);

3) Competitive profit. The objective is to guarantee a profit, which is defined as "value - cost", competitive to the omniscient single-price auction. 
We aim to solve the above problem by generalizing the random sampling and profit extraction paradigm of competitive auctions (Goldberg, Hartline, and Wright 2001). The contributions of our work can be summarized as follows:

1) We enrich the class of competitive auctions by considering a more general optimization objective and a more general demand valuation function.

2) We propose a truthful, individually rational and profit competitive mechanism for our setting. The profit obtained by the mechanism is constant-competitive for fixed markets and $(8+\epsilon)$-competitive for large markets.

3) We propose a more natural approach to model and solve the application problem of cellular traffic offloading. As it is a rather general framework, it can also be used for other similar problems.

\section{Related Work}

Most of the pioneering studies on designing incentive mechanisms for cellular traffic offloading, e.g., (Zhuo et al. 2011; Paris et al. 2013; Dong et al. 2014; Zhuo et al. 2014), focus on the settings where the underlying traffic or offloading demands are known to the CSP or can be estimated precisely and efficiently, and the optimization objective is social welfare maximization. Basically, for these settings the celebrated Vickrey-Clarke-Groves (VCG) mechanism (Vickrey 1961; Clarke 1971; Groves 1973) is a perfect solution.

However, mechanism design with optimization objectives/constraints relates to payments, e.g., revenue maximization, profit maximization (Hartline and Karlin 2007), and so on, are traditionally well-known hard problems. As far as we know, such objectives are relatively new in current cellular traffic offloading study. Although Myerson's work in the 1980's (Myerson 1981) has solved the revenue maximization problem in Bayesian single-parameter case, how to design the optimal mechanism for the general case still remains a widely open problem. However, there have been some noteworthy progress. In the past two decades, researchers showed that several important subclasses of optimal mechanism design problems can be solved by using technologies from computer science even in the prior-free case, for example, competitive auctions (Goldberg, Hartline, and Wright 2001), budget-feasible mechanisms (Singer 2010) and so on. The problem of maximizing CSP's capacity gain under the constraint of a strict budget was firstly studied by (Zhang et al. 2016) and (Wu et al. 2019).

The research of competitive auctions stems from (Goldberg, Hartline, and Wright 2001; Goldberg et al. 2006). Basically, subsequent work can be categorized into two classes: designing auctions with improved competitive ratios, e.g., (Fiat et al. 2002; Hartline and Mcgrew 2005; Ichiba and Iwama 2010; Chen, Gravin, and Lu 2014); or further exploring the space of solvable prior-free optimal mechanism design problem based on the methodology of random sampling, e.g., (Fiat et al. 2002; Cary et al. 2008; Abrams 2006; Bei et al. 2012). Our work intrinsically belongs to the later class.

The first study that brought multi-unit 2-parameter auction into the scope of random sampling mechanisms is the multi-unit budget-constrained auction problem studied in (Borgs et al. 2005), in which there are a limited number of identical goods, each bidder has a private unit valuation and a private overall hard budget. They designed for it an asymptotically competitive auction with the competitive ratio tends to 1 as the bidder dominance (defined in section 5.3) tends to 0 . However, this mechanism performs badly when the bidder dominance is large. (Abrams 2006) proposed another random sampling mechanism to solve this problem, improved the competitive ratio for cases with high bidder dominance. Later, in the study of cellular traffic offloading, (Zhang et al. 2016) showed that (Abrams 2006)'s mechanism can be easily adapted to a multi-unit procurement auction setting, where the auctioneer (cellular service provider) aims to maximize the amount of service purchased from the agents (femtocells) while the total payment is strictly bounded within a given budget. Our work is mainly motivated by (Zhang et al. 2016). We generalize the demand valuation function from additive to LDR and focus on a different optimization objective, i.e., profit. We propose a framework to generate competitive profit in multi-unit procurement auctions. Note that (Zhang et al. 2016)'s mechanism belongs to the class of budget feasible mechanisms (Singer 2010; Chen, Gravin, and Lu 2011; Bei et al. 2012; Chan and Chen 2014; Biswas et al. 2015), especially the subclass of multiunit budget feasible mechanisms, which have been systematically studied in (Chan and Chen 2014). Thus our setting also closely relates to the settings studied in (Chan and Chen 2014), but is different in both the optimization objective and the number of private parameters. We are also aware of the fact that (Ray, Mandal, and Narahari 2015) have studied a similar setting of profit maximizing multi-unit procurement auction. However, they use different techniques to design the profit extractor, and in the 2-parameter case, their current results only apply to linear demand valuation functions.

\section{Preliminaries}

\section{Cellular Traffic Offloading}

In cellular traffic offloading the CSP gains extra network capacity by purchasing offloading resources (or service) from $\alpha$ agents in the macrocell sector, denoted as $[\alpha]=\{1, \ldots, \alpha\}$. We assume each agent $i \in[\alpha]$ has

- a capacity $\hat{m}_{i} \in \mathbb{N}$, representing the maximal number of resource units she can provide, and

- a unit cost $\hat{c}_{i} \in \mathbb{R}^{+}$, representing her cost of providing each resource unit.

The resource units owned by each agent $i \in[\alpha]$ can be denoted as $(i, 1),(i, 2), \ldots,\left(i, \hat{m}_{i}\right)$, where $(i, j)$ refers to agent $i$ 's $j$-th unit. Note that, femtocells have a much smaller coverage compared with macrocell base stations, offloading resources purchased from one femtocell is not able to handle all traffic from the entire macrocell. To deal with this practical issue, we can divide the entire sector into $\beta$ nonoverlapping regions, denoted as $[\beta]=\{1, \ldots, \beta\}$, each of which is fully covered by the femtocells that reside in it. So from a region's point of view, there is no difference between the units purchased from different agents inside it. 
We denote an allocation as $\mathcal{A}=\left(a_{1}, \ldots, a_{n}\right)$ where each $a_{i} \in\left\{0,1, \ldots, \sigma_{i}\right\}$ is the number of units obtained from agent $i$. The capacity gain of a region $j \in[\beta]$, denoted as $s_{j}(\mathcal{A})$, equals to the total units purchased within this region. We can let $r(i)$ denote the region agent $i$ belongs to, and then the agents in region $j$ can be denoted as $R_{j}=\{i \in$ $[\alpha]: r(i)=j\}$. Hence, we have $s_{j}(\mathcal{A})=\sum_{i \in R_{j}} a_{i}$.

For each region $j$, we can specify a weight $w_{j} \in R^{+}$, which intuitively represents the value obtained by using a resource unit in this region to offload some data traffic. So, as the number of units obtained in a region increases, the marginal value of the $i$ th unit is $w_{j} \cdot \operatorname{Pr}\left(d_{j} \geq i\right)$, where $d_{j}$ is a random variable representing the resource demand in region $j$, and $\operatorname{Pr}\left(d_{j} \geq i\right)$ is the probability that the $i$ th unit will be used. We denote $\operatorname{Pr}\left(d_{j} \geq i\right)$ as $\delta_{i}^{j}$. Therefore, given an allocation $\mathcal{A}$, the obtained value in region $j$ is

$$
v_{j}(\mathcal{A})=\sum_{i=1}^{s_{j}(\mathcal{A})} w_{j} \delta_{i}^{j}=w_{j} \cdot \sum_{i=1}^{s_{j}(\mathcal{A})} \delta_{i}^{j}
$$

where $1 \geq \delta_{1}^{j} \geq \delta_{2}^{j} \geq \delta_{3}^{j} \geq \cdots \geq 0$ is a sequence of nonnegative real numbers upper bounded by 1 (we assume they are given in advance).

The value of allocation $\mathcal{A}$, denoted as $v(\mathcal{A})$, is the sum of the values obtained in all regions, i.e.,

$$
v(\mathcal{A})=\sum_{j=1}^{\beta} \sum_{i=1}^{s_{j}(\mathcal{A})} w_{j} \delta_{i}^{j}=\sum_{j=1}^{\beta} w_{j} \sum_{i=1}^{s_{j}(\mathcal{A})} \delta_{i}^{j}
$$

Given an allocation $\mathcal{A}=\left(a_{1}, a_{2}, \ldots, a_{i}, \ldots, a_{n}\right)$, adding one extra resource unit $(i, j)$ of agent $i$ will result in a new allocation $\mathcal{A}+e_{i}=\left(a_{1}, a_{2}, \ldots, a_{i}+1, \ldots, a_{n}\right)$, in which the expected offloading in the system increases by $m_{\mathcal{A}}(i, j)=\delta_{s_{r(i)}(\mathcal{A})+1}^{r(i)}$ in region $r(i)$, and the value will increase by $v\left(\mathcal{A}+e_{i}\right)-v(\mathcal{A})=m_{\mathcal{A}}(i, j) \cdot w_{r(i)}$, which we refer to as unit $(i, j)$ 's marginal value. It is easy to check that, for any $\mathcal{A}$ and $\mathcal{A}^{\prime}$ such that $a_{i} \leq a_{i}^{\prime}$ for each $i$, and for any agent $j$,

$$
\begin{aligned}
v\left(\mathcal{A}+e_{j}\right)-v(\mathcal{A}) & =\delta_{s_{r(j)}(\mathcal{A})+1}^{r(j)} \cdot w_{r(j)} \geq \delta_{s_{r(j)}\left(\mathcal{A}^{\prime}\right)+1}^{r(j)} \cdot w_{r(j)} \\
& =v\left(\mathcal{A}^{\prime}+e_{j}\right)-v\left(\mathcal{A}^{\prime}\right)
\end{aligned}
$$

According to the the definitions in (Chan and Chen 2014), $v(\cdot)$ belongs to the diminishing return class. It actually forms a proper subset of the diminishing return class, since each unit's marginal value is only locally depended on the allocation in her region. We call this valuation class as local diminishing return (LDR) functions, formally, it is a diminishing return function where the agents are partitioned into disjointed sets, and for any allocation $\mathcal{A}$ and $\mathcal{A}^{\prime}$, and any agent $j$, we have $v\left(\mathcal{A}+e_{j}\right)-v(\mathcal{A})=v\left(\mathcal{A}^{\prime}+e_{j}\right)-v\left(\mathcal{A}^{\prime}\right)$ if $a_{i}=a_{i}^{\prime}$ for all $i$ such that $r(i)=r(j)$. If the payments to the agents are $\mathcal{T}=\left(t_{1}, \ldots, t_{n}\right)$, where $t_{i} \in \mathbb{R}^{+}$, then the profit $\pi(\mathcal{A}, \mathcal{T})$ is the difference between the obtained value and the total payments to the agents, i.e.,

$$
\pi(\mathcal{A}, \mathcal{T})=v(\mathcal{A})-\sum_{i=1}^{\alpha} t_{i}=\sum_{j=1}^{\beta}\left(\sum_{i=1}^{s_{j}(\mathcal{A})} w_{j} \delta_{i}^{j}-\sum_{i \in R_{j}} t_{i}\right)
$$

Clearly, the item in the above bracket is actually the profit obtained in region $j$, and so $\pi(\mathcal{A}, \mathcal{T})$ equals to the profits obtained in all the regions added together. We aim to find out the allocation and payment that maximizes the profit. Since the valuations in the regions are independent from each other, the following result trivially holds.

Proposition 1. Allocation and payment $(\mathcal{A}, \mathcal{T})$ maximizes the profit iff it maximizes the profit obtained in each region.

Therefore, our problem reduces to finding out the optimal allocation and payment for each region.

\section{Economic model}

For an arbitrary region there is a set of agents (bidders) $[n]=\{1, \ldots, n\}$, and the agents compete for providing their resource units to the auctioneer. The actual values of $\hat{m}_{i}$ and $\hat{c}_{i}$ are only privately known by each agent $i$ herself, and we refer to $\tau_{i}=\left(\hat{m}_{i}, \hat{c}_{i}\right)$ as agent $i$ 's type. The auctioneer runs a single round sealed bid procurement auction with all the agents. As a seller, each agent $i$ submits a bid $\boldsymbol{b}_{i}=\left(m_{i}, c_{i}\right)$ to the auctioneer. We use $\hat{\boldsymbol{m}}$ and $\hat{\boldsymbol{c}}$ to denote the capacity profile $\left(\hat{m}_{1}, \cdots, \hat{m}_{n}\right)$ and unit cost profile $\left(\hat{c}_{1}, \cdots, \hat{c}_{n}\right)$ respectively, and use $\boldsymbol{m}$ and $\boldsymbol{c}$ to denote the corresponding capacity bid profile $\left(m_{1}, \cdots, m_{n}\right)$ and unit cost bid profile $\left(c_{1}, \cdots, c_{n}\right)$ respectively. Based on the bid vector $\boldsymbol{b}=\left(\boldsymbol{b}_{1}, \cdots, \boldsymbol{b}_{n}\right)$, the auctioneer determines the outcome using a predefined mechanism $\mathcal{M}=(\boldsymbol{x}, \boldsymbol{p})$ consists of

1) an allocation function $x_{i}: \boldsymbol{b}_{1} \times \cdots \times \boldsymbol{b}_{n} \rightarrow \mathbb{N}$, and

2) a payment function $p_{i}: \boldsymbol{b}_{1} \times \cdots \times \boldsymbol{b}_{n} \rightarrow \mathbb{R}^{+}$.

That is, after receiving the vector $\boldsymbol{b}$, for each $i$, the auctioneer tries to purchase $x_{i}(\boldsymbol{b})$ units of goods from agent $i$, and gives agent $i$ a payment of $p_{i}(\boldsymbol{b})$ if succeed, a punishment as serious as possible otherwise. So the utility of agent $i$ is

$$
u_{i}(\boldsymbol{b})=\left\{\begin{array}{rr}
p_{i}(\boldsymbol{b})-c_{i} \cdot x_{i}(\boldsymbol{b}) & x_{i}(\boldsymbol{b}) \leq m_{i} \\
-\infty & x_{i}(\boldsymbol{b})>m_{i}
\end{array}\right.
$$

Note that, such a utility function can be realized by (Nisan and Ronen 2001)'s framework of mechanism with verification, but we omit the details to avoid unnecessary complexity. We assume the agents are rational in the sense that they always pursue higher utilities, and the agents do not collude and have complete knowledge of the auction mechanism.

The auctioneer's valuation to the purchased unit set $S$ is actually a symmetric submodular function (Singer 2010), when $|S|=j(j \in \mathbb{N})$, the auctioneer obtains the value: $v_{\boldsymbol{r}}(j)=\sum_{i=1}^{j} r_{i}$, where $r_{i}=w_{i} \delta_{i}$ is the $i$-th unit's marginal value, $\boldsymbol{r}=\left\langle r_{1}, \ldots, r_{M}\right\rangle$ is a non-increasing sequence of non-negative real numbers, $M$ is a sufficiently large natural number. We refer to $v_{\boldsymbol{r}}$ as the local demand valuation function. Given a mechanism $(\boldsymbol{x}, \boldsymbol{p})$ and an arbitrary bid vector $\boldsymbol{b}$, the profit of the auctioneer is

$\pi_{\boldsymbol{r}}(\boldsymbol{b})=v_{\boldsymbol{r}}\left(\sum_{j=1}^{n} x_{j}(\boldsymbol{b})\right)-\sum_{j=1}^{n} p_{j}(\boldsymbol{b})=\sum_{i=1}^{\sum_{j=1}^{n} x_{j}(\boldsymbol{b})} r_{i}-\sum_{j=1}^{n} p_{j}(\boldsymbol{b})$ 


\section{Regional Optimal Omniscient Auctions}

In the omniscient case, the capacity $\hat{m}_{i}$ and unit cost $\hat{c}_{i}$ of each bidder $i$ are assumed to be known by the auctioneer. Given the demand valuation function $v_{\boldsymbol{r}}(\cdot)$, the optimal multi-price omniscient auction $\mathcal{O P} \mathcal{T}_{\boldsymbol{r}}^{m}$ can be realized by greedily buying the lowest cost unit $(i, j)$ with a price equals to its $\operatorname{cost} \hat{c}_{i}$ until the marginal value becomes lower than the unit cost, breaking ties lexicographically. We denote the cost of the $i$ th lowest cost unit as $q_{i}$, and denote the number of selected items as $k_{m}$, then the obtained profit is:

$$
P_{\boldsymbol{r}}^{m}(\hat{\boldsymbol{m}}, \hat{\boldsymbol{c}})=\max _{j} \sum_{i=1}^{j}\left(r_{i}-q_{i}\right)=\sum_{i=1}^{k_{m}}\left(r_{i}-q_{i}\right)
$$

The clear price adopted by the optimal single-price omniscient auction $\mathcal{O} \mathcal{P} \mathcal{T}_{r}^{s}$ must just equal to the highest unit cost of all the winner agents. Therefore, the profit obtained by $\mathcal{O P} \mathcal{T}_{r}^{s}$ is:

$$
P_{\boldsymbol{r}}^{s}(\hat{\boldsymbol{m}}, \hat{\boldsymbol{c}})=\max _{j} \sum_{i=1}^{j}\left(r_{i}-q_{j}\right)=\sum_{i=1}^{k_{s}}\left(r_{i}-q_{k_{s}}\right)
$$

where $k_{s}$ is the number of selected units.

Lemma 2. $P_{\boldsymbol{r}}^{s}(\hat{\boldsymbol{m}}, \hat{\boldsymbol{c}}) \geq \frac{1}{\ln k_{m}+\mathcal{O}(1)} P_{\boldsymbol{r}}^{m}(\hat{\boldsymbol{m}}, \hat{\boldsymbol{c}})$.

Proof. The optimality of $P_{\boldsymbol{r}}^{s}(\hat{\boldsymbol{m}}, \hat{\boldsymbol{c}})$ follows

$\forall j: \sum_{i=1}^{k_{s}}\left(r_{i}-q_{k_{s}}\right) \geq \sum_{i=1}^{j}\left(r_{i}-q_{j}\right) \geq \sum_{i=1}^{j}\left(r_{j}-q_{j}\right)=j\left(r_{j}-q_{j}\right)$

Accordingly, $\forall j:\left(r_{j}-q_{j}\right) \leq \frac{1}{j} \sum_{i=1}^{k_{s}}\left(r_{i}-q_{k_{s}}\right)$. So, by equation 8 we can further obtain

$P_{\boldsymbol{r}}^{m}(\hat{\boldsymbol{m}}, \hat{\boldsymbol{c}}) \leq \sum_{i=1}^{k_{m}} \frac{1}{j} \sum_{i=1}^{k_{s}}\left(r_{i}-q_{k_{s}}\right) \leq\left[\ln k_{m}+\mathcal{O}(1)\right] \cdot P_{\boldsymbol{r}}^{s}(\hat{\boldsymbol{m}}, \hat{\boldsymbol{c}})$

Therefore, lemma 1 holds.

Thus, $\mathcal{O} \mathcal{P} \mathcal{T}_{r}^{s}$ can be guaranteed to obtain a $\frac{1}{\mathcal{O}(1)+\ln k_{m}}$ sized fragment of the $\mathcal{O P} \mathcal{T}_{r}^{m}$ profit.

\section{A Regional Truthful Profit Extractor}

\section{Feasible Clear Price and Purchasing Quota}

First of all, the agents are divided into two partitions randomly. Given a partition, we can first reorder the agents by increasing unit cost bid, break ties randomly, and then all the $N=\sum_{i=1}^{n} m_{i}$ units can be seen as having been ordered lexicographically in a queue $\mathcal{Q}$. Let $S_{i}$ denote the set of units from the first $i$ agents, and so we have $\left|S_{i}\right|=\sum_{j=1}^{i} m_{j}$. This setting can be depicted graphically in figure 1, where the $x$ axis represents the number of the units, and the $y$-axis represents unit cost or price. The units of each agent are depicted as a rectangle and placed in order on the $x$-axis. The height and width of each rectangle represent the unit cost and capacity of the corresponding agent respectively. Making a profit of $R$ ultimately reduces to determining a feasible combination $(X, P)$, with which we can buy $X$ units from the agents at the unit price $P$ and obtain profit $R$.
- We call $X \in \mathbb{N}$ as a feasible (purchasing) quota if there is a $P_{X} \in \mathbb{R}^{+}$such that $\left(X, P_{X}\right)$ is a feasible combination;

- We call $P \in \mathbb{R}^{+}$as a feasible clear price if there is a $X_{P} \in \mathbb{N}$ such that $\left(X_{P}, P\right)$ is a feasible combination.

Note that, since individual rationality must be guaranteed, at a clear price of $P$ we can only buy units from agents that bid unit costs no higher than $P$. For an arbitrary $(X, P)$, we call it as a possible combination if and only if

$$
\sum_{i=1}^{X} r_{i}-P X=R
$$

It is straightforward to verify the following result.

Proposition 3. $(X, P)$ is a feasible combination iff $(X, P)$ is a possible combination and there are at least $X$ units whose bid costs don't exceed P.

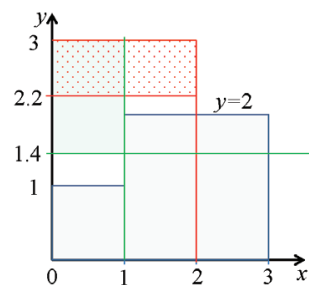

Figure 1: An illustration for example 1

Example 1. Consider a partition with 2 agents: agent 1 with capacity 1 and unit cost 1 , agent 2 with capacity 2 and unit cost 2 . If $R=1.6, r_{1}=r_{2}=3, \forall i \geq 3: r_{i}=0$ and all the agents truthfully bid their types, then both $\left(X_{1}, P_{1}\right)=$ $(1,1.4)$ and $\left(X_{2}, P_{2}\right)=(2,2.2)$ are feasible combinations.

1) Given a mechanism which adopts the clear price $P_{1}=$ 1.4 to buy the first $X_{1}=1$ unit in the queue $\mathcal{G}$ (i.e., the unit of agent 1 ), agent 1 's utility will be 0.4 . Actually, agent 1 can obtain a higher utility by bidding her unit cost higher at 1.5: In this case, $\left(X_{1}, P_{1}\right)$ becomes infeasible, and $P_{2}=2.2$ will be adopted to buy 1 unit from agent 1 and 1 unit from agent 2 , and so agent 1 's utility becomes 1.2 .

2) To avoid the above issue, we can consider always adopting the feasible combination with the highest feasible clear price. That is, $\left(X_{2}, P_{2}\right)$ will be adopted. But this still isn't good enough, since agent 2 can misreport a unit cost lower than 1, and then she will switch her position with agent 1 and thus sells 2 units at the unit price 2.2, making her utility doubled. Fortunately, the above issue can be avoided by purchasing from the winner agents in a random order.

Example 1 implies adopting a non-highest feasible clear price may sometimes motivate misreporting. So, among all the feasible combinations we always choose the one containing the highest feasible clear price. Now, we define:

$$
X^{*}=\min \left\{j \in[N]: \sum_{i=1}^{j}\left(r_{i}-r_{j+1}\right) \geq R\right\}
$$




$$
P^{*}=\frac{\sum_{i=1}^{X^{*}} r_{i}-R}{X^{*}}
$$

Actually the search space can be constricted to $\left[X^{*}\right]$ :

Lemma 4. If both $(X, P)$ and $\left(X^{\prime}, P^{\prime}\right)$ are possible, then

1) if $X \leq X^{\prime} \leq X^{*}$, then $P \leq P^{\prime} \leq P^{*}$;

2) if $X^{*} \leq X^{\prime} \leq X$, then $P^{*} \geq P^{\prime} \geq P$;

3) if $P<P^{\prime}, X \leq X^{*}$ and $X^{\prime} \leq X^{*}$ then $X<X^{\prime}$.

Proof. From Equations 13 and 14, it follows:

$$
r_{1} \geq \cdots \geq r_{X^{*}}>P^{*} \geq r_{X^{*}+1} \geq \cdots \geq r_{N}
$$

1) According to inequality $15, r_{i}-P^{*}>0$ for all $i \leq X^{*}$. Therefore, from equation 12 , we can derive

$$
R=\sum_{i=1}^{X^{\prime}}\left(r_{i}-P^{\prime}\right)=\sum_{i=1}^{X^{*}}\left(r_{i}-P^{*}\right) \geq \sum_{i=1}^{X^{\prime}}\left(r_{i}-P^{*}\right)
$$

and it immediately follows that $P^{\prime} \leq P^{*}$. So, $r_{i}-P^{\prime}>0$ for all $i \leq X^{\prime}$, and for the same reason, we can further obtain $P \leq \bar{P}^{\prime}$. Therefore, $P \leq P^{\prime} \leq P^{*}$.

2) According to equation $15, r_{i}-P^{*} \leq 0$ for all $X^{*}+1 \leq$ $i \leq N$. So, equation 16 also holds, and it follows $P^{\prime} \leq P^{*}$. Therefore, $\sum_{i=1}^{X^{*}}\left(r_{i}-P^{\prime}\right) \geq R$, so

$$
0 \geq \sum_{i=X^{*}+1}^{X^{\prime}}\left(r_{i}-P^{\prime}\right) \geq\left(X^{\prime}-X^{*}\right)\left(r_{X^{\prime}}-P^{\prime}\right)
$$

Accordingly, $r_{X^{\prime}} \leq P^{\prime}$, and it implies $r_{i}-P^{\prime} \leq 0$ for all $X^{\prime}+1 \leq i \leq N$. Therefore, similarly we can obtain $P \leq P^{\prime}$, and finally derive $P^{*} \geq P^{\prime} \geq P$.

3) From equation 12 , we can obtain

$$
R=\sum_{i=1}^{X}\left(r_{i}-P\right)=\sum_{i=1}^{X^{\prime}}\left(r_{i}-P^{\prime}\right)
$$

Since $r_{i}-P>0$ for all $i \leq X$, and $r_{i}-P^{\prime}>0$ for all $i \leq X^{\prime}, P \leq P^{\prime}$ in equation 18 implies $X \leq X^{\prime}$.

Corollary 5. If $P$ is a feasible clear price then $P \leq P^{*}$.

Proof. It follows directly from lemma 4.

Intuitively, for all the possible combinations $(X, P)$, as $X$ gradually increases from 0 to $N, P$ monotonously increases until $X=X^{*}$, afterward $P$ monotonously decreases. In this sense, we refer to $X^{*}$ as the turning point.

Lemma 6. If $X$ is the largest feasible purchasing quota in $\left[X^{*}\right]$, then $P=\frac{\sum_{i=1}^{X} r_{i}-R}{X}$ is the highest feasible clear price.

Proof. Let $X$ be the largest feasible purchasing quota in $\left[X^{*}\right]$. Assume that there is another feasible $X^{\prime}$ s.t. $P^{\prime}=$ $\frac{\sum_{i=1}^{X^{\prime}} r_{i}-R}{X^{\prime}}>P$. There are only 2 cases, as shown in the following:

Case $1\left(X^{\prime} \leq X^{*}\right)$ : Since $X^{\prime}<X \leq X^{*}$, and from lemma 4, it follows $P \geq P^{\prime}$. Contradiction!
Case $2\left(X^{\prime}>X^{*}\right)$ : Since $X^{\prime}$ is feasible, there are at least $X^{\prime}$ units whose cost bids don't exceed $P^{\prime}$. By lemma $5, P^{\prime} \leq$ $P^{*}$, so the number of units whose cost bids don't exceed $P^{*}$ is more than $X^{*}$, and therefore $P^{*}$ is a feasible clear price. So, $P=P^{*} \geq P^{\prime}$, and this is also a contradiction.

Finally, we can further constrict the search space to several special points according to the following result.

Lemma 7. If $X$ is a largest feasible purchasing quota in $\left[X^{*}\right]$, then $X \in\left\{X^{*}\right\} \cup\left\{\left|S_{i}\right|:\left|S_{i}\right|<X^{*}\right\}$.

Proof. Assume that there is a $X$ being a largest feasible purchasing quota, satisfying $\left|S_{i-1}\right|<X<\left|S_{i}\right|$. By proposition 3 , we have $c_{1} \leq \cdots \leq c_{i} \leq \frac{\sum_{i=1}^{X} r_{i}-R}{X}$. By lemma 4, $X<\left|S_{i}\right|<X^{*}$, it follows $\frac{\sum_{i=1}^{X} r_{i}-R}{X} \leq \frac{\sum_{i=1}^{\left|S_{i}\right|} r_{i}-R}{\left|S_{i}\right|}$. So, $\left|S_{i}\right|$ is also a feasible purchasing quota, and $X$ can't be the largest feasible purchasing quota. This is a contradiction. With the same approach, we can show that any $X$ satisfying $\max \left\{\left|S_{i}\right|:\left|S_{i}\right|<X^{*}\right\}<X<X^{*}$ is also impossible.

\section{The proposed profit extractor}

Based on the results from the previous subsection, we can now design a truthful profit extractor, as follows.

\section{Mechanism PROFITEXTRACT $r, R$}

Input: $n^{\prime}$ agents with bid $(\boldsymbol{m}, \boldsymbol{c})$

1. Reorder the agents by increasing unit cost, break ties randomly and reorder $\boldsymbol{m}$ and $\boldsymbol{c}$ accordingly;

2. $N^{\prime} \leftarrow \sum_{i=1}^{n^{\prime}} m_{i}$;

if $\sum_{i=1}^{N^{\prime}} r_{i}<R$ then return;

$I \leftarrow\left\{j \in\left[N^{\prime}\right]: \sum_{i=1}^{j}\left(r_{i}-r_{j+1}\right) \geq R\right\}$;

if $I=\emptyset$ then $X^{*} \leftarrow N$; else $X^{*} \leftarrow \min I$;

$\ell \leftarrow \min \left\{j \in\left[n^{\prime}\right]: \sum_{i=1}^{j} r_{i} \geq X^{*}\right\}$;

3. if $\sum_{i=1}^{X^{*}}\left(r_{i}-c_{\ell}\right) \geq R$ then $X \leftarrow X^{*}$;

$P \leftarrow \frac{\sum_{i=1}^{X} r_{i}-R}{X}$

else end

$T \leftarrow\left\{j \in[\ell-1]: c_{j} \leq \frac{\sum_{i=1}^{\left|S_{j}\right|} r_{i}-R}{\left|S_{j}\right|}\right\} ;$

if $T=\emptyset$ then $X \leftarrow 0 ; P \leftarrow 0$;

else $\ell^{*} \leftarrow \max T ; X \leftarrow\left|S_{\ell^{*}}\right| ; P \leftarrow \frac{\sum_{i=1}^{X} r_{i}-R}{X} ;$

4. BuY-In-RANDOM-ORder $\left(\left[n^{\prime}\right], X, P\right)$;

Function BUY-IN-RANDOM-ORDER

Input: agent set $S$, clear price $P$, purchasing quota $X$

1. $W \leftarrow S$;

2. while $X \neq 0$ do

Select an agent $i$ from $W$ randomly and delete it; if $c_{i} \leq P$ then

Select $X^{\prime}=\min \left\{X, m_{i}\right\}$ units from agent $i$; Pay $P \cdot X^{\prime}$ units of money to agent $i$; $X \leftarrow X-X^{\prime}$

end end 
As shown in figure 2, firstly (in step 1), the mechanism reorders the agents by increasing unit cost bid, break ties randomly. Next, (in step 2) it tries to find out the turning point $X^{*}$ and agent $\ell$ who owns the $X^{*}$-th unit. Afterward, (in step 3) it tries to find the largest element $X$ which is feasible in $\left\{X^{*}\right\} \cup\left\{\left|S_{i}\right|:\left|S_{i}\right|<X^{*}\right\}$. According to lemma 7, $X$ must be the largest feasible purchasing amount in $\left[X^{*}\right]$; and according to lemma $6, P$ must be the largest feasible clear price; finally, (in step 4) it considers the agents in random order (the necessity has been demonstrated by example 1), and try to buy $X$ units in total at unit price $P$. Note that, we call all the agents with unit cost bids at most $P$ as winner agents, since all such agents have some chance to sell.

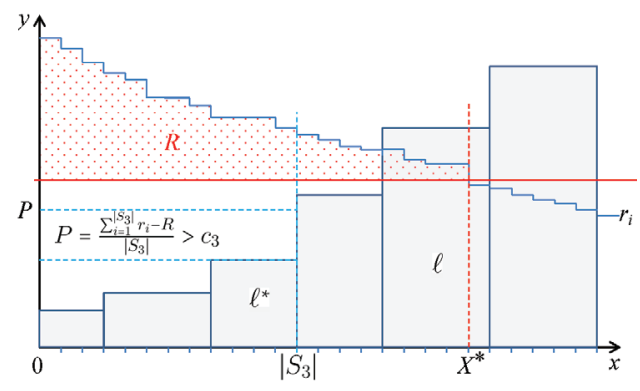

Figure 2: Basic idea of the profit extractor

With respect to truthfulness, since each agent $i$ has two private parameters, i.e., $\hat{m}_{i}$ and $\hat{c}_{i}$, we must prove that no agent can obtain a higher expected utility by combinatorially misreporting $\hat{m}_{i}$ and $\hat{c}_{i}$, despite what other agents bid.

Lemma 8. If $(X, P)$ is a feasible combination, and $X=$ $\left|S_{i}\right|<X^{*}$ is a largest feasible quota, then $P<c_{i+1}$.

Proof. Assume that $P \geq c_{i+1}$. Consider the possible combination $\left(X^{\prime}, P^{\prime}\right)$, where $X^{\prime}=\min \left\{\left|S_{i+1}\right|, X^{*}\right\}$. By lemma 4(1), $X^{\prime}>X$ implies $P^{\prime}>P \geq c_{i+1}$. So, the number of units with bid costs less than $P^{\prime}$ is at least $\left|S_{i+1}\right|$. According to proposition $3, X^{\prime}>X$ is also feasible. This is obviously a contradiction.

\section{Theorem 9. PROFITEXTRACT $r, R$ is truthful.}

Proof. For an arbitrary agent $i$ with capacity $\hat{m}_{i}$ and unit cost $\hat{c}_{i}$, if other agents have bid $\left(\boldsymbol{m}_{-i}, \boldsymbol{c}_{-i}\right)$, her utility will be determined by her bid. Let $X, P, U_{i}$ be the purchasing quota, clear price and agent $i$ 's utility, respectively, when agent $i$ truthfully bid $\left(\hat{m}_{i}, \hat{c}_{i}\right)$, and assume they become $X^{\prime}, P^{\prime}, U_{i}^{\prime}$ respectively, when agent $i$ bid $\left(m_{i}^{\prime}, c_{i}^{\prime}\right)$ instead. There are 2 cases in total as shown in the following:

Case $1\left(X=X^{*}\right): P=P^{*}$, and by proposition 3 , the total number of units the winner agents have is no less than $X^{*}$. So, the number of units each winner agent sell is a random variable. The randomness comes from the order by which the agents are considered.

(1.1) Agent $i$ is a loser: We have $\mathbb{E}\left[U_{i}\right]=0$ and $\hat{c}_{i}>P^{*}$. $\mathbb{E}\left[U_{i}^{\prime}\right]>\mathbb{E}\left[U_{i}\right]=0$ requires $P^{\prime}>\hat{c}_{i}$, and therefore $P^{\prime}>$ $P^{*}$. But according to corollary 5 , this is impossible.

(1.2) Agent $i$ is a winner: $\mathbb{E}\left[U_{i}\right]>0$, and there are the following possibilities: $(a)$ if agent $i$ bid $c_{i}^{\prime}>P$ then it will be excluded from the winner set and obtain a lower expected utility $\mathbb{E}\left[U_{i}^{\prime}\right]=0$. Since according to corollary 5 , there is no feasible clear price which is higher than $P^{*} ;(b)$ if agent $i$ bid $m_{i}^{\prime}=\hat{m}_{i}$ and $c_{i}^{\prime} \leq P$, then by proposition $3,\left(X^{*}, P^{*}\right)$ is still feasible, the winner set is the same and $P^{\prime}=P$. So, $\mathbb{E}\left[U_{i}^{\prime}\right]=\mathbb{E}\left[U_{i}\right] .(c)$ if agent $i$ bid $m_{i}^{\prime}>\hat{m}_{i}$ and $c_{i}^{\prime} \leq P$, $\left(X^{*}, P^{*}\right)$ is still feasible and we have $P^{\prime}=P$. If $X^{*} \leq$ $\hat{m}_{i}, \mathbb{E}\left[U_{i}\right]=\mathbb{E}\left[U_{i}^{\prime}\right]$, else $\mathbb{E}\left[U_{i}^{\prime}\right]=-\infty$. (d) if agent $i$ bid $m_{i}^{\prime}<\hat{m}_{i}$ and $c_{i}^{\prime} \leq P$ : if $X^{\prime}=X$ and $P^{\prime}=P$, then the winner set is the same, and $\mathbb{E}\left[U_{i}^{\prime}\right]<\mathbb{E}\left[U_{i}\right]$, else $X^{\prime}<X$, and by lemma $4, P^{\prime}<P$. We let $\Delta=\Omega-X^{*}$, where $\Omega$ is the total number of units of the winner agents when agent $i$ bid truthfully. It is clear that agent $i$ has to at least bid $\Delta+1$ units lower for $X^{*}$ to become infeasible. So, we have $m_{i}^{\prime}<\hat{m}_{i}-\Delta$, and $\mathbb{E}\left[U_{i}^{\prime}\right]<\left(\hat{m}_{i}-\Delta\right)\left(P^{\prime}-\hat{c}_{i}\right)<$ $\left(\hat{m}_{i}-\Delta\right)\left(P-\hat{c}_{i}\right)<\mathbb{E}\left[U_{i}\right]$. Note that, the last " $<$ " above is due to the fact that the worst case for $U_{i}$ is when agent $i$ is the last to sell her units, and in this case agent $i$ sells $\hat{m}_{i}-\Delta$ unit at unit price $P$.

Case $2\left(X<X^{*}\right): X \in\left\{\left|S_{i}\right|:\left|S_{i}\right|<X^{*}\right\}$, and according to lemma 8 , every winner agent sells all the units she has bid, so there is no uncertainty.

(2.1) Agent $i$ is a loser: $P<\hat{c}_{i}$ and $\mathbb{E}\left[U_{i}\right]=0$. To obtain a higher utility, it must be $P^{\prime}>\hat{c}_{i}$, and $P^{\prime}>P$, by lemma 4(3) it follows $X^{\prime}>X$. So, agent $i$ must have higher bid her capacity. Since $m_{i}<X^{\prime}, \mathbb{E}\left[U_{i}^{\prime}\right]=-\infty$. Contradiction!

(2.2) Agent $i$ is a winner: $\mathbb{E}\left[U_{i}\right]=\left(P-\hat{c}_{i}\right) \cdot \hat{m}_{i}$ and $\mathbb{E}\left[U_{i}^{\prime}\right]=\left(P^{\prime}-\hat{c}_{i}\right) \cdot \mathbb{E}\left[X_{i}^{\prime}\right]$ where $\mathbb{E}\left[X_{i}^{\prime}\right]$ is the expected number of units agent $i$ sells. Assume that $\mathbb{E}\left[U_{i}^{\prime}\right]>\mathbb{E}\left[U_{i}\right]$, i.e., $\left(P^{\prime}-\hat{c}_{i}\right) \cdot \mathbb{E}\left[X_{i}\right]>\left(P-\hat{c}_{i}\right) \cdot \hat{m}_{i}$. It is obvious that $\mathbb{E}\left[X_{i}\right] \leq \hat{m}_{i}$, since otherwise agent $i$ must sometimes sell more than her capacity $\hat{m}_{i}$, so $\mathbb{E}\left[U_{i}\right]=-\infty$. Thus, it must be $P^{\prime}>P$, and it will result in a contradiction just as above.

So, truthfulness holds in the sense that for each agent, the expected utility is maximized by bidding her capacity and unit cost truthfully, despite what the other agents bid.

Afterward, individual rationality follows directly from the specification of the proposed profit extraction mechanism.

Theorem 10. PROFITEXTRACT $\boldsymbol{r}, R$ is individually rational.

Proof. For each agent $i$, if she bids $\left(m_{i}, c_{i}\right)$ and the clear price $P \leq c_{i}$, then at most $m_{i}$ items will be purchased from her at the price $P$. By theorem 9, every agent will bid truthfully, so the utility of every agent must be at least 0 .

Finally, we can show that, the proposed profit extractor can always obtain any reasonable target profit $R$, which is lower than the omniscient single-price auction profit.

Lemma 11. The mechanism PROFITEXTRACT $\boldsymbol{r}, R$ obtains profit $R$ if $P_{\boldsymbol{r}}^{s}(\hat{\boldsymbol{m}}, \hat{\boldsymbol{c}}) \geq R$ and 0 otherwise.

Proof. If $P_{\boldsymbol{r}}^{s}(\hat{\boldsymbol{m}}, \hat{\boldsymbol{c}}) \geq R$, then by equation $9, \sum_{i=1}^{k_{s}}\left(r_{i}-\right.$ $\left.q_{k_{s}}\right) \geq R$ where $k_{s} \in[N]$. So there is a $P \geq q_{k_{s}}$ satisfying $\sum_{i=1}^{k_{s}}\left(r_{i}-P\right)=R$, so $\left(k_{s}, P\right)$ is a feasible combination. Therefore, at least PROFITEXTRACT $\boldsymbol{r}, R$ can adopt $\left(k_{s}, P\right)$ to obtain a profit of $R$. If $P_{\boldsymbol{r}}^{s}(\boldsymbol{m}, \boldsymbol{c})<R$, then $\sum_{i=1}^{k}\left(r_{i}-\right.$ 
$\left.q_{k}\right)<R$ for all $k \in[N]$, so there is no feasible combination, thus PROFITEXTRACT $\boldsymbol{r}, R$ will obtain profit 0 .

\section{The Profit competitive mechanism}

Finally, we can propose the following mechanism, which is adopted in each region.

Mechanism PROFIT-PA $r$

Input: $n$ agents with bid $(\boldsymbol{m}, \boldsymbol{c})$

1. Partition the agents u.a.r. into two sets $\Sigma_{A}$ and $\Sigma_{B}$ with bids $\left(\boldsymbol{m}^{\prime}, \boldsymbol{c}^{\prime}\right)$ and $\left(\boldsymbol{m}^{\prime \prime}, \boldsymbol{c}^{\prime \prime}\right)$ respectively.

2. $R_{A} \leftarrow P_{r}^{s}\left(\boldsymbol{m}^{\prime}, \boldsymbol{c}^{\prime}\right)$;

$R_{B} \leftarrow P_{r}^{s}\left(\boldsymbol{m}^{\prime \prime}, c^{\prime \prime}\right)$

3. With prob. $\frac{1}{2}$ run PROFITEXTRACT $\boldsymbol{r}, R_{B}\left(\boldsymbol{m}^{\prime}, \boldsymbol{c}^{\prime}\right)$; With prob. $\frac{1}{2}$ run PROFITEXTRACT $\boldsymbol{r}, R_{A}\left(\boldsymbol{m}^{\prime \prime}, \boldsymbol{c}^{\prime \prime}\right)$;

This mechanism firstly divide the agents into 2 partitions randomly (step 1), then the $\mathcal{O} \mathcal{P} \mathcal{T}_{r}^{s}$ profit of each partition is computed (step 2), and finally one of the partition's $\mathcal{O P} \mathcal{T}_{r}^{s}$ profit is used as target to generate profit from the other partition (step 3). It is trivially computationally tractable, individually rational and truthful.

Theorem 12. PROFIT-PA $r$ is truthful.

Proof. For each agent, either she will be in a partition where the utility is 0 despite how she bids, or she will take part in an auction specified by PROFITEXTRACT $r, R$ with a target profit independent of her bid. Since PROFITEXTRACT $\boldsymbol{r}, R$ is truthful, each agent's best choice is to bid truthfully.

Lower bound The bidder dominance factor (Abrams 2006) is defined as $\alpha=\frac{k_{s}}{\max \left\{\tilde{m}_{1}, \ldots, \tilde{m}_{n}\right\}}$, in which $k_{s}$ is the total number of units purchased by $\mathcal{O P} \mathcal{T}_{r}^{s}, \tilde{m}_{i}(1 \leq i \leq n)$ is agent $i$ 's contribution, so actually $k_{s}=\sum_{i=1}^{n} \tilde{m}_{i}$.

Theorem 13. PROFIT-PA $r$ is $\frac{8 \alpha}{\alpha-1}$-competitive to $\mathcal{O P} \mathcal{T}_{\boldsymbol{r}}^{s}$.

Proof. Let $\Sigma^{s}$ be the agents who contributes goods to $\mathcal{O P} \mathcal{T}_{r}^{s}$ and $P_{s}$ is the adopted clear price. Let $\Sigma_{A}^{s}=\Sigma^{s} \cap \Sigma_{A}$ and $\Sigma_{B}^{s}=\Sigma^{s} \cap \Sigma_{B}$. We denote $k^{\prime}=\sum_{i \in \Sigma_{A}^{s}} \tilde{m}_{i}$ and $k^{\prime \prime}=\sum_{i \in \Sigma_{B}^{s}} \tilde{m}_{i}$, so $k^{\prime}+k^{\prime \prime}=k_{s}$. Moreover, it must be $P_{\boldsymbol{r}}^{s}\left(\boldsymbol{m}^{\prime}, \boldsymbol{c}^{\prime}\right) \geq \sum_{i=1}^{k^{\prime}}\left(r_{i}-P_{s}\right)$, since in this case, at least we can obtain a profit of buying $k^{\prime}$ items at the price $P_{s}$. Similarly, $P_{\boldsymbol{r}}^{s}\left(\boldsymbol{m}^{\prime \prime}, \boldsymbol{c}^{\prime \prime}\right) \geq \sum_{i=1}^{k^{\prime \prime}}\left(r_{i}-P_{s}\right)$. By Lemma 11 , only when $\min \left\{P_{\boldsymbol{r}}^{s}\left(\boldsymbol{m}^{\prime}, \boldsymbol{c}^{\prime}\right), P_{\boldsymbol{r}}^{s}\left(\boldsymbol{m}^{\prime \prime}, \boldsymbol{c}^{\prime \prime}\right)\right\}$ is adopted as target, the profit extractor can succeed, and in this case (with prob. $\left.\geq \frac{1}{2}\right)$ :

$$
\begin{aligned}
\pi(\boldsymbol{m}, \boldsymbol{c}) & =\min \left\{P_{\boldsymbol{r}}^{s}\left(\boldsymbol{m}^{\prime}, \boldsymbol{c}^{\prime}\right), P_{\boldsymbol{r}}^{s}\left(\boldsymbol{m}^{\prime \prime}, \boldsymbol{c}^{\prime \prime}\right)\right\} \\
& \geq \min \left\{\sum_{i=1}^{k^{\prime}}\left(r_{i}-P_{s}\right), \sum_{i=1}^{k^{\prime \prime}}\left(r_{i}-P_{s}\right)\right\} \\
& \geq \frac{\min \left\{k^{\prime}, k^{\prime \prime}\right\}}{k_{s}} \cdot \sum_{i=1}^{k_{s}}\left(r_{i}-P_{s}\right)
\end{aligned}
$$

and for all other cases, we have $\pi(\boldsymbol{m}, \boldsymbol{c})=0$. Moreover, according to the results of (Abrams 2006), we have

$$
\mathbb{E}\left[\min \left\{k^{\prime}, k^{\prime \prime}\right\}\right] \geq \frac{\alpha-1}{4 \alpha} k_{s}
$$

Based on equations 19 and 20, we can further obtain:

$$
\begin{aligned}
\mathbb{E}[\pi(\boldsymbol{m}, \boldsymbol{c})] & \geq \frac{1}{2} \cdot \frac{\mathbb{E}\left[\min \left\{k^{\prime}, k^{\prime \prime}\right\}\right]}{k_{s}} \cdot P_{\boldsymbol{r}}^{s}(\hat{\boldsymbol{m}}, \hat{\boldsymbol{c}}) \\
& \geq \frac{\alpha-1}{8 \alpha} P_{\boldsymbol{r}}^{s}(\hat{\boldsymbol{m}}, \hat{\boldsymbol{c}})
\end{aligned}
$$

Hence, the expected profit is at least $\frac{\alpha-1}{8 \alpha} P_{\boldsymbol{r}}^{s}(\hat{\boldsymbol{m}}, \hat{\boldsymbol{c}})$.

In a fixed market, $\alpha$ is a constant and therefore the competitive ratio is a constant. In a small market where only 1 agent's good is purchased by the omniscient single-price auction, we have $\alpha=1$, and in large markets where single agent's contribution is trivial, we have $\alpha \rightarrow+\infty$. Thus as the market starts from a small one and grows larger and larger, the competitive ratio starts from $+\infty$ and gradually converges to 8 . The following corollary follows.

Corollary 14. PROFIT-PA $r$ is $(8+\epsilon)$-competitive to $\mathcal{O P} \mathcal{T}_{r}^{s}$ in large markets.

Upper bound With respect to the best possible performance of the mechanism, it is easy to see it is upper bounded by the $\mathcal{O P} \mathcal{T}_{r}^{m}$ performance, and we can further show this bound is tight.

Proposition 15. There are cases with type profile $(\hat{\boldsymbol{m}}, \hat{\boldsymbol{c}})$ and valuation function $v_{\boldsymbol{r}}$ satisfying $\pi(\hat{\boldsymbol{m}}, \hat{\boldsymbol{c}})=\frac{1}{1+\epsilon}$. $P_{\boldsymbol{r}}^{m}(\hat{\boldsymbol{m}}, \hat{\boldsymbol{c}})$

Proof. Consider the following case: there are $n$ agents, each of which can provide 1 item at a price $x$, and $r_{1}=v, \forall i \geq$ $2: r_{i}=0$, where $v \geq x$. The omniscient multi-price auction $\mathcal{O P} \mathcal{T}_{r}^{m}$ will purchase 1 item at the price $x$, and obtain the profit equals to $v-x$, while PROFIT-PA $r$ will extract the profit $v-x$ from one of the 2 partitions if both of them are nonempty, otherwise it will return a profit equals to 0 , and the expected profit is $\frac{n-1}{n+1}(v-x)$. Therefore when the number of agents is large enough the above result holds.

Note that, in the above proof, if we buy from both partitions, the expected profit will become $\frac{n-1}{n+1}(v-2 x)$, and this means when $v \leq 2 x$ the expected profit is unbounded low. We actually circumvent this issue by always buying from at most one partition.

\section{Conclusion}

We model cellular traffic offloading as problem of maximizing the profit of a multi-unit procurement auction with symmetric submodular demand valuation function in the priorfree case. We solve this problem using the framework of competitive analysis. The challenges we have faced are how to design a truthful profit extractor which can be used to guarantee any reasonable profit and how to correlate the two sub-markets formed by random sampling to achieve a guaranteed global performance. We propose a computationally tractable, truthful, individually rational and profit competitive mechanism for this setting. The profit obtained by our 
mechanism is consistently competitive (to the omniscient optimal single-price auction) for fixed markets and $(8+\epsilon)$ competitive for large markets.

\section{Acknowledgments}

This paper was supported by the National Key Research and Development Program of China (Grant Nos. 2017YFD0401001, 2018YFB1403400), the National Natural Science Foundation of China (Grant Nos. 91646204, 61876080, 71871109), the Key Research and Development Program of Jiangsu Province (Grant No. BE2019105), and the Collaborative Innovation Center of Novel Software Technology and Industrialization at Nanjing University.

\section{References}

Abrams, Z. 2006. Revenue maximization when bidders have budgets. In Proc. of 17th ACM-SIAM Symp. on Discrete Algorithm (SODA-06), 1074-1082.

Balasubramanian, A.; Mahajan, R.; and Venkataramani, A. 2010. Augmenting mobile $3 \mathrm{~g}$ using wifi. In Proc. of Int. Conf. on Mobile Systems, Applications, and Services, 209-222.

Bei, X.; Chen, N.; Gravin, N.; and Lu, P. 2012. Budget feasible mechanism design:from prior-free to bayesian. In Proc. of 44th ACM Symp. on Theory of Computing (STOC-12), 449-458.

Biswas, A.; Jain, S.; Mandal, D.; and Narahari, Y. 2015. A truthful budget feasible multi-armed bandit mechanism for crowdsourcing time critical tasks. In Proc. of Int. Conf. on Autonomous Agents and Multiagent Systems (AAMAS-15), 11011109.

Borgs, C.; Chayes, J.; Immorlica, N.; Mahdian, M.; and Saberi, A. 2005. Multi-unit auctions with budget-constrained bidders. In Proc. of ACM Conf. on Electronic Commerce (EC-05), 4451.

Cary, M. C.; Flaxman, A. D.; Hartline, J. D.; and Karlin, A. R. 2008. Auctions for structured procurement. In Proc. of 19th ACM-SIAM Symp. on Discrete Algorithms (SODA-08), 304313.

Chan, H., and Chen, J. 2014. Truthful multi-unit procurements with budgets. In Proc. of Workshop on Internet and Network Economics (WINE-14), 89-105.

Chen, N.; Gravin, N.; and Lu, P. 2011. On the approximability of budget feasible mechanisms. In Proc. of 22th ACMSIAM Symp. on Discrete Algorithms (SODA-11), volume 27, 685-699.

Chen, N.; Gravin, N.; and Lu, P. 2014. Optimal competitive auctions. In Proc. of 46th ACM Symp. on Theory of Computing (STOC-14), 253-262.

Cisco. 2019. Cisco visual networking index: Global mobile data traffic forecast update, 2017-2022 white paper. https://www.cisco.com/c/en/us/solutions/collateral/serviceprovider/visual-networking-index-vni/white-paper-c11738429.html. [Online; Updated February-2019].

Clarke, E. H. 1971. Multipart pricing of public goods. Public Choice 11(11):17-33.

Dong, W.; Rallapalli, S.; Jana, R.; Qiu, L.; Ramakrishnan, K. K.; Razoumov, L.; Zhang, Y.; and Cho, T. W. 2014. ideal:incentivized dynamic cellular offloading via auctions. IEEE/ACM Trans. on Networking 22(4):1271-1284.
Fiat, A.; Goldberg, A. V.; Hartline, J. D.; and Karlin, A. R. 2002. Competitive generalized auctions. In Proc. of ACM Symp. on Theory of Computing (STOC-02), 72-81.

Goldberg, A. V.; Hartline, J. D.; Karlin, A. R.; Saks, M.; and Wright, A. 2006. Competitive auctions. Games \& Economic Behavior 55(2):242-269.

Goldberg, A. V.; Hartline, J. D.; and Wright, A. 2001. Competitive auctions and digital goods. In Proc. of 12th ACM-SIAM Symp. on Discrete Algorithms (SODA-08), 735-744.

Groves, T. 1973. Incentives in teams. Econometrica 41:617631.

Hartline, J., and Karlin, A. 2007. Algorithmic Game Theory. Cambridge University Press. chapter Profit maximization in mechanism design, 331-361.

Hartline, J. D., and Mcgrew, R. 2005. From optimal limited to unlimited supply auctions. In Proc. of ACM Conf. on Electronic Commerce (EC-05), 175-182.

Ichiba, T., and Iwama, K. 2010. Averaging techniques for competitive auctions. In Proc. of Meeting on Algorithm Engineering and Experiments, 74-81.

Myerson, R. B. 1981. Optimal auction design. Mathematics of Operations Research 6(1):58-73.

Nisan, N., and Ronen, A. 2001. Algorithmic mechanism design. Games \& Economic Behavior 35(1-2):166-196.

Paris, S.; Martisnon, F.; Filippini, I.; and Lin, C. 2013. A bandwidth trading marketplace for mobile data offloading. In Proc. of IEEE Int. Conf. on Computer Communications (INFOCOM13), 430-434.

Ray, A.; Mandal, D.; and Narahari, Y. 2015. Profit maximizing prior-free multi-unit procurement auctions with capacitated sellers. In Proc. of Int. Conf. on Autonomous Agents and Multiagent Systems (AAMAS-15), 1753-1754.

Singer, Y. 2010. Budget feasible mechanisms. In Proc. of IEEE Symp. on Foundations of Computer Science (FOCS-10), 765-774.

Vickrey, W. 1961. Counterspeculation, auctions, and competitive sealed tenders. Journal of Finance 16(1):8-37.

Wang, W.; Wu, X.; Xie, L.; and Lu, S. 2015. Femto-matching: Efficient traffic offloading in heterogeneous cellular networks. In Proc. of IEEE Int. Conf. on Computer Communications (INFOCOM-15), 325-333.

Wu, J.; ; Zhang, Y.; Qiao, Y.; Zhang, L.; Wang, C.; and Xie, J. 2019. Multi-unit budget feasible mechanisms for cellular traffic offloading. In Proc. of Int. Conf. on Autonomous Agents and Multiagent Systems (AAMAS-19), 1693-1701.

Zhang, Y.; Tang, S.; Chen, T.; and Zhong, S. 2016. Competitive auctions for cost-aware cellular traffic offloading with optimized capacity gain. In Proc. of IEEE Int. Conf. on Computer Communications (INFOCOM-16), 1-9.

Zhuo, X.; Gao, W.; Cao, G.; and Dai, Y. 2011. Win-coupon: An incentive framework for $3 \mathrm{~g}$ traffic offloading. In Proc. of IEEE Int. Conf. on Network Protocols (ICNP-11), 206-215.

Zhuo, X.; Gao, W.; Cao, G.; and Hua, S. 2014. An incentive framework for cellular traffic offloading. IEEE Trans. on Mobile Computing 13(3):541-555. 